\title{
Barriers to pandemic influenza vaccination and uptake of seasonal influenza vaccine in the post-pandemic season in Germany
}

Merle M Böhmer ${ }^{1,4,5^{*}}$, Dietmar Walter ${ }^{1,4}$, Gerhard Falkenhorst ${ }^{1}$, Stephan Müters ${ }^{3}$, Gérard Krause ${ }^{2}$ and Ole Wichmann ${ }^{1}$

\begin{abstract}
Background: In Germany, annual vaccination against seasonal influenza is recommended for certain target groups (e.g. persons aged $\geq 60$ years, chronically ill persons, healthcare workers (HCW)). In season 2009/10, vaccination against pandemic influenza $\mathrm{A}(\mathrm{H} 1 \mathrm{~N} 1)$ pdm09, which was controversially discussed in the public, was recommended for the whole population. The objectives of this study were to assess vaccination coverage for seasonal (seasons 2008/09-2010/11) and pandemic influenza (season 2009/10), to identify predictors of and barriers to pandemic vaccine uptake and whether the controversial discussions on pandemic vaccination has had a negative impact on seasonal influenza vaccine uptake in Germany.
\end{abstract}

Methods: We analysed data from the 'German Health Update' (GEDA10) telephone survey $(n=22,050)$ and a smaller GEDA10-follow-up survey $(n=2,493)$, which were both representative of the general population aged $\geq 18$ years living in Germany.

Results: Overall only 8.8\% of the adult population in Germany received a vaccination against pandemic influenza. High socioeconomic status, having received a seasonal influenza shot in the previous season, and belonging to a target group for seasonal influenza vaccination were independently associated with the uptake of pandemic vaccines. The main reasons for not receiving a pandemic vaccination were 'fear of side effects' and the opinion that 'vaccination was not necessary'. Seasonal influenza vaccine uptake in the pre-pandemic season 2008/09 was 52.8\% among persons aged $\geq 60$ years; $30.5 \%$ among HCW, and $43.3 \%$ among chronically ill persons. A decrease in vaccination coverage was observed across all target groups in the first post-pandemic season 2010/11 (50.6\%, $25.8 \%$, and $41.0 \%$ vaccination coverage, respectively).

Conclusions: Seasonal influenza vaccination coverage in Germany remains in all target groups below 75\%, which is a declared goal of the European Union. Our results suggest that controversial public discussions about safety and the benefits of pandemic influenza vaccination may have contributed to both a very low uptake of pandemic vaccines and a decreased uptake of seasonal influenza vaccines in the first post-pandemic season. In the upcoming years, the uptake of seasonal influenza vaccines should be carefully monitored in all target groups to identify if this trend continues and to guide public health authorities in developing more effective vaccination and communication strategies for seasonal influenza vaccination.

Keywords: Vaccination, Influenza, Coverage, Pandemic, Germany

\footnotetext{
* Correspondence: BoehmerM@rki.de

${ }^{1}$ Immunization Unit, Robert Koch Institute, Berlin, Germany

${ }^{4}$ Charité - University Medicine Berlin, Berlin, Germany

Full list of author information is available at the end of the article
} 


\section{Background}

In Germany, annual influenza epidemics usually occur during the winter months December to March. In the last decade, an estimated zero to 19,000 excess deaths per year were attributable to influenza virus infections [1]. Moreover, approximately one to six million influenza-related excess physician consultations per season were estimated for Germany [2]. Severe influenza virus infections or influenza-related complications typically occur in the very young and elderly population as well as in persons with underlying chronic medical conditions.

Annual vaccination has proven to be an effective method to reduce the burden of influenza disease [3]. In Germany, vaccination against seasonal influenza is recommended by the Standing Committee on Vaccination (STIKO) for individuals who have either an increased risk to develop severe influenza disease (i.e. persons aged $\geq 60$ years, pregnant women, and persons with certain chronic medical conditions) or who are likely to transmit the virus to vulnerable groups (e.g. health care workers $(\mathrm{HCW})$ ) [4]. Vaccination is free of charge for the target groups in Germany. During the influenza pandemic 2009/10, STIKO additionally recommended vaccination with a monovalent vaccine against the pandemic influenza virus strain $A(H 1 N 1) p d m 09$ for the whole population. Due to expected limitations in vaccine supplies at the beginning of the vaccination campaign, STIKO defined and ranked priority groups for the pandemic vaccination: 1) HCW, 2) persons with underlying chronic conditions, 3) pregnant women, 4) household contacts of vulnerable persons, 5) all other persons aged 6 months to 24 years, 6) all other persons aged 25-59 years, 7) all other person aged 60 years and above [5]. The pandemic vaccination campaign started in Germany on 26 October 2009 [6]. The AS03adjuvanted monovalent vaccine Pandemrix ${ }^{\circledR}$ was almost exclusively used and available in sufficient quantities [7].

During the pandemic, vaccination against $\mathrm{A}(\mathrm{H} 1 \mathrm{~N} 1)$ pdm09 was subject to controversial discussions, not only in Germany but also in many other countries worldwide [7-9]. Main topics of the debate in the media and among experts and 'self-proclaimed experts' were vaccine safety, effectiveness, and concern that there were too little data on the new vaccines or vaccine ingredients (especially new adjuvants) available. Moreover, the general necessity of vaccination in view of the relative mildness of the pandemic influenza disease was called into question [7,9-11]. As a result, compliance with the national recommendations for pandemic vaccination was very poor in Germany. Since Germany has no central immunization registry, information on vaccination coverage (and factors influencing coverage) is only available from telephone and household surveys [12-15]. According to the results of thirteen consecutive crosssectional telephone surveys (total $n=13,010$ ) conducted during the pandemic, only $8.1 \%$ of the general population aged $\geq 14$ years living in Germany received a vaccine against pandemic influenza [6].

To develop target group specific communication strategies and to enhance compliance with the official recommendations it is important to monitor vaccine uptake in each of the target groups and to understand factors that influence uptake. This applies not only for annual influenza vaccination campaigns but also for the planning of future vaccination campaigns during a pandemic. The influence of the 2009/2010 pandemic situation on seasonal influenza vaccine uptake in Germany in the post-pandemic seasons has so far not been investigated. For this purpose, we utilized data from the large $(\sim 22,000$ respondents) 'German Health Update 2010' (GEDA10) telephone survey and a smaller GEDA10 follow-up survey ( $\sim 2,500$ respondents). The objectives of our study were (1) to assess seasonal influenza vaccination coverage for seasons $2008 / 09$ to $2010 / 11$, (2) to assess pandemic influenza vaccination coverage for season $2009 / 10$, (3) to identify predictors of and barriers to pandemic vaccine uptake, and (4) to detect a potential influence of the pandemic situation on seasonal influenza vaccine uptake in the first post-pandemic season (2010/11).

\section{Methods}

\section{Study population and survey design}

The GEDA survey design has been described previously $[12,13,16]$. In brief, GEDA is a large annual telephone survey which is conducted by the Robert Koch Institute (RKI) as a part of Germany's national health monitoring. The study population consists of persons $\geq 18$ years of age who are living in a private household in Germany, have sufficient knowledge of the German language, and can be contacted via landline telephone. The GEDA study protocol was approved by Germany's federal and regional data-protection commissioners. All data were collected and analysed in an anonymous manner.

In this study we present data from GEDA10 which was conducted between 22 September 2009 and 10 July 2010. Since the annual GEDA survey was not conducted in 2010/2011, we conducted a follow-up interview among a subsample of 2.493 GEDA10 respondents (from now on referred to as the GEDA follow-up survey) from 1 April to 2 July 2011 to assess seasonal influenza vaccine uptake for the post pandemic season 2010/11. Based on a sample size calculation, 385 subjects were needed to estimate a prevalence of 50\% ("worst case scenario") vaccine uptake with a confidence interval of $+/-5 \%$. Our sample size of $\sim 2,400$ subjects for the followup survey was based on the premise to estimate a prevalence (i.e. vaccination coverage) of $50 \%$ for up to six subgroups (=cells; $6 * 385 \approx 2400$ ). 
Since both surveys were conducted by RKI, the ownership of the data lies with RKI and we did not have to obtain permission to use the data for this study. Similar to the previous GEDA survey (GEDA09), a public use file of the GEDA10 dataset will be provided soon. Data from the GEDA follow-up survey will not be openly available.

To control for possible selection biases, weighting factors for the GEDA10 sample were constructed by taking age, sex, educational status, geographical region, and household size into consideration. Potential participants of the follow-up survey were sampled disproportionally to their weighting factors in GEDA10. We applied this method to avoid that groups which were already underrepresented in GEDA10 become underrepresented again at the sampling stage in the follow-up sample and thus to prevent further bias. Weighting factors for the followup survey were constructed in a first step on the basis of the values calculated for GEDA10 (for age, sex, education, adipositas, smoking status, subjective health, physical activity, employment status), and, in a second step, on population data gathered in the Microcensus 2008 [17], taking geographical region, age, sex, and educational status into account.

Information on seasonal influenza vaccination status for season 2008/2009 was collected from all participants of GEDA10. Between 1 January 2010 and 10 July 2010 respondents were additionally asked to provide information on seasonal and pandemic influenza vaccination status for season 2009/10. Unvaccinated survey participants of GEDA10 were additionally asked to state their reasons for not receiving a pandemic influenza vaccination. Information on seasonal vaccination status for season 2010/11 was collected from all respondents of the GEDA follow-up survey. In the follow-up survey, respondents were additionally asked by whom they were vaccinated (general practitioner/other physician in private practice/occupational physician/other) and in which month they received the vaccination against seasonal influenza in season 2010/11. One should note that GEDA10 does not cover the paediatric population (aged 0-17 years) for which pandemic vaccination was also recommended in Germany. Information on vaccination coverage in this particular age-group was therefore not available from this data source.

We calculated the response for GEDA10 by using Response Rate 3 as defined by the American Association for Public Opinion Research (AAPOR) [18]. Response Rate 3 is the proportion of the number of complete interviews divided by the number of interviews plus the number of non-interviews (refusal and break-off plus non-contacts plus others) plus cases of unknown eligibility. For cases of unknown eligibility Response Rate 3 estimates what proportion of cases of unknown eligibility is actually eligible. This estimation is based on the proportion of eligible households among all numbers for which a definitive determination of status was obtained (hence a very conservative estimate). We additionally calculated the cooperation rate at respondent level, which is defined as the proportion of all respondents interviewed of all respondents ever contacted [18]. Since the GEDA follow-up survey was not a random digit dialling study (as GEDA10), we reported the minimal response rate (Response Rate 1 as defined by AAPOR, [18]) for the follow-up survey.

\section{Definition of variables}

Socio-economic status levels were created as described by Lampert and Kroll on the basis of self-reported educational, income, and professional status of survey respondents [19]. In accordance with the STIKOrecommendations [4], persons were classified into the target groups for seasonal influenza vaccination in our study if they reported (1) to be $\geq 60$ years of age, (2) to have at least one underlying chronic disease (defined here as having a chronic underlying respiratory, cardiovascular, liver, or renal disease, cancer, or diabetes), or (3) to work as HCW. Since female respondents of childbearing age were not asked whether they had been pregnant during the last influenza season, it was not possible to include pregnant women as target group in our analysis. The geographic region category 'Western Federal States' (WFS) comprised the federal states SchleswigHolstein, Bremen, Hamburg, Lower Saxony, Hesse, Rhineland-Palatinate, Saarland, North Rhine-Westphalia, Baden-Württemberg and Bavaria; 'Eastern Federal States' (EFS) comprised Mecklenburg-Vorpommern, Brandenburg, Berlin, Saxony-Anhalt, Thuringia and Saxony.

\section{Statistical analysis}

Data analysis was performed using PASW 18.0 for Windows (SPSS Inc., Chicago, USA). Proportions were calculated by using procedures for the analysis of complex samples. Univariate analyses were conducted to determine associations between pandemic influenza vaccine uptake and socio-demographic, health-related and professional factors. A p-value $\leq 0.05$ was considered to indicate a statistically significant difference. Odds ratios (OR) and 95\% confidence intervals (CI) were calculated as appropriate. Multivariable analysis was performed by entering variables potentially associated with vaccine uptake ( $\mathrm{p}$-value $<0.2$ in univariate analysis) into a multivariable logistic regression model in a first step, followed by step-wise backward removal of variables with a p-value $>0.05$ to produce a final model. Interaction terms were included to account for effect modification between independent variables. 


\section{Results}

\section{Sample characteristics}

In total, 22,050 telephone interviews were conducted during the study period of GEDA10 and 2,493 participants were re-interviewed for the follow-up survey. An overview of the survey populations is given in Table 1. The median age was 48.0 years (range 18-99 years) in GEDA10 and 49.7 years (range 19-96 years) in the follow-up survey. Response Rate 3 was $28.9 \%$ in GEDA10; the cooperation rate at respondent level was 55.8\%. Response Rate 1 was $75.0 \%$ in the follow up survey.

\section{Seasonal influenza vaccination coverage}

Information on seasonal influenza vaccination status was available for over $99.8 \%$ in each of the study samples for the three seasons under investigation (seasons 2008/092010/11). Vaccination coverage for the three seasons by sex, age group, place of residence, and target group is presented in Table 2. To allow comparability with international studies, seasonal influenza vaccine uptake among $\geq 65$ year-olds was additionally calculated and revealed a coverage of $56.1 \%$ (95\% CI: $54.0-58.2)$ in season 2008/09, 50.2\% (95\% CI: 47.5-52.9) in season 2009/ 10, and 54.2\% (95\% CI: 48.4-59.8) in season 2010/11. For influenza season 2009/10, vaccination coverage was calculated by age-groups in decades (Figure 1). Vaccine uptake in both the target population for seasonal influenza

Table 1 Characteristics of participants in the 'German Health Update Survey' (GEDA10) and the GEDA10 follow-up survey

\begin{tabular}{|c|c|c|c|c|}
\hline & \multicolumn{2}{|c|}{$\begin{array}{c}\text { GEDA10 } \\
\text { September } 2009 \text { - } \\
\text { July } 2010\end{array}$} & \multicolumn{2}{|c|}{$\begin{array}{c}\text { Follow-up } \\
\text { survey } \\
\text { April - July } 2011\end{array}$} \\
\hline & $n^{*}$ & $\%^{*}$ & $\mathrm{n}^{*}$ & $\%{ }^{*}$ \\
\hline Total & 22,050 & 100 & 2,493 & 100 \\
\hline \multicolumn{5}{|l|}{ Sex } \\
\hline female & 11,347 & 51.5 & 1,287 & 51.6 \\
\hline male & 10,703 & 48.5 & 1,206 & 48.4 \\
\hline \multicolumn{5}{|l|}{ Age group } \\
\hline $18-39$ years & 7,145 & 32.4 & 761 & 30.5 \\
\hline $40-59$ years & 8,142 & 36.9 & 937 & 37.6 \\
\hline$\geq 60$ years & 6,763 & 30.7 & 795 & 31.9 \\
\hline \multicolumn{5}{|l|}{ Place of residence } \\
\hline Western Federal States & 17,433 & 79.1 & 1,968 & 79.0 \\
\hline Eastern Federal States & 4,617 & 20.9 & 525 & 21.0 \\
\hline \multicolumn{5}{|l|}{ Underlying chronic disease } \\
\hline yes & 7,260 & 32.9 & 972 & 39.0 \\
\hline no & 14,790 & 67.1 & 1,521 & 61.0 \\
\hline \multicolumn{5}{|l|}{ Health care worker } \\
\hline yes & 1,117 & 5.1 & 225 & 9.0 \\
\hline no & 20,933 & 94.9 & 2,268 & 91.0 \\
\hline
\end{tabular}

vaccination (defined here as persons who have an underlying chronic disease, or work as HCW) and non-target population increased with age and was highest in persons $\geq 70$ years. The vast majority $(97.8 \%)$ of vaccinated persons had received their influenza vaccination for season 2010/11 by the end of December 2010.

Figure 2 shows trends in seasonal influenza vaccine uptake among the three different target groups and the non-target group for four consecutive seasons (2007/08 to 2010/11; results for season 2007/08 according to a previously published analysis of GEDA 2009 data [12]). While vaccination coverage slightly decreased in persons aged $\geq 60$ years and in persons with underlying chronic diseases between seasons 2007/08 and 2009/10, there was an increase in vaccine uptake among $\mathrm{HCW}$ from season $2007 / 08$ to $2008 / 09$. In all subgroups under investigation, vaccine uptake for season 2009/10 was higher in the follow-up sample (empty symbols) compared to the GEDA10 sample (filled symbols). Considering only the results from the follow-up survey (Figure 2), a significant decrease in seasonal influenza vaccination coverage between seasons 2009/10 and 2010/11 was observed for persons with underlying chronic conditions $(\mathrm{p}=0.04), \mathrm{HCWs}(\mathrm{p}=0.03)$, and persons not targeted for seasonal influenza vaccination $(\mathrm{p}<0.01)$. For persons $\geq 60$ years of age there was also a decrease, but without reaching statistical significance. In season 2010/11, $83.4 \%$ of respondents who received a seasonal influenza shot were vaccinated by their general practitioner, $4.7 \%$ by another physician in private practice (e.g. gynaecologist, paediatrician), $9.4 \%$ by an occupational physician, $0.4 \%$ by a hospital physician, and $2.1 \%$ by any other physician. Of those who were vaccinated by a general practitioner or by an occupational physician, $18.4 \%$ and $59.4 \%$ did not belong to a target group, respectively.

\section{Pandemic influenza vaccination coverage}

Information on pandemic influenza vaccine uptake was available for $99.9 \%$ of the respective study population in GEDA $10(n=13,048)$. Vaccination coverage by sex, age group, place of residence, socio-economic status and different target groups for seasonal influenza vaccination is presented in Table 3. In total, 8.8\% (95\% CI: 8.2-9.5) of the general adult population in Germany received a vaccination against pandemic influenza. With $11.2 \%$ (95\% CI: 10.2-12.3) pandemic vaccine uptake was significantly higher in persons belonging to the target group for seasonal influenza vaccination as compared to the non-seasonal influenza target group (6.4\%; 95\% CI: 5.7-7.1; $\mathrm{p}<0.001)$.

The most frequently reported reasons for not receiving the vaccination were (1) 'fear of side effects of pandemic vaccines' (stated by 37.2\%; 95\% CI: 36.1-38.3), (2) 'pandemic vaccination is not necessary' (33.8\%; 95\% CI: 32.7-34.9), (3) 'pandemic vaccination not officially recommended 
Table 2 Seasonal influenza vaccine uptake by sex, age group, place of residence and target group, seasons 2008/09 - 2010/11, Germany

\begin{tabular}{|c|c|c|c|}
\hline & Season $2008 / 09$ & Season $2009 / 10$ & Season $2010 / 11$ \\
\hline & $\overline{n=22,009 \%(95 \% \mathrm{Cl})^{*}}$ & $\overline{n=13,040 \%(95 \% \mathrm{Cl})^{*}}$ & $\overline{n=2,492 \%(95 \% \mathrm{Cl})^{*}}$ \\
\hline Total & $29.8(29.0-30.6)$ & $26.6(25.6-27.6)$ & $28.3(26.0-30.6)$ \\
\hline \multicolumn{4}{|l|}{ Sex } \\
\hline female & $30.8(29.7-31.9)$ & $27.2(25.8-28.6)$ & $28.2(25.3-31.3)$ \\
\hline male & $28.8(27.6-30.0)$ & $26.0(24.5-27.5)$ & $28.3(25.0-32.0)$ \\
\hline \multicolumn{4}{|l|}{ Age group } \\
\hline 18-39 years & $14.8(13.8-15.9)$ & $12.8(11.6-14.1)$ & $13.3(10.7-16.6)$ \\
\hline $40-59$ years & $23.9(22.8-25.0)$ & $21.2(19.8-22.6)$ & $21.5(18.6-24.7)$ \\
\hline$\geq 60$ years & $52.8(51.0-54.5)$ & $47.5(45.2-49.8)$ & $50.6(45.7-55.5)$ \\
\hline \multicolumn{4}{|l|}{ Place of residence } \\
\hline Western Federal States & $26.7(25.8-27.6)$ & $24.0(22.9-25.1)$ & $25.8(23.3-28.4)$ \\
\hline Eastern Federal States & $41.5(39.6-43.5)$ & $36.7(34.3-39.2)$ & $37.7(32.6-43.0)$ \\
\hline \multicolumn{4}{|l|}{ Target group } \\
\hline health care workers & $30.5(27.6-33.6)$ & $27.3(23.6-31.3)$ & $25.8(20.4-32.1)$ \\
\hline chronically ill persons & $43.3(41.7-44.9)$ & $39.8(37.8-41.9)$ & $41.0(36.9-45.3)$ \\
\hline non-target group & $16.6(15.8-17.5)$ & $14.2(13.2-15.3)$ & $14.8(12.5-17.5)$ \\
\hline
\end{tabular}

*Weighted data.

for me' (16.6\%; 95\% CI: 15.8-17.5), and (4) 'reject vaccinations in general' (8.5\%; 95\% CI: 7.8-9.2).

\section{Factors associated with pandemic influenza}

\section{vaccine uptake}

Results of univariate and multivariable analysis of factors potentially associated with pandemic influenza vaccine uptake are shown in Table 3. Having received a seasonal influenza vaccination in the previous season (season 2008/09) was the strongest independent predictor of pandemic influenza vaccination. However, this effect differed by age group and we therefore included an interaction term in the final model. Additionally, working as $\mathrm{HCW}$, having a chronic disease, high socioeconomic status, and being male were significantly associated with higher uptake in multivariable analysis.

\section{Discussion}

The aim of this study was to assess the uptake of seasonal influenza vaccines in specific target groups for seasons 2008/09 and 2009/10, as well as for pandemic influenza vaccines during the pandemic season 2009/10 in Germany in the total adult population by using data

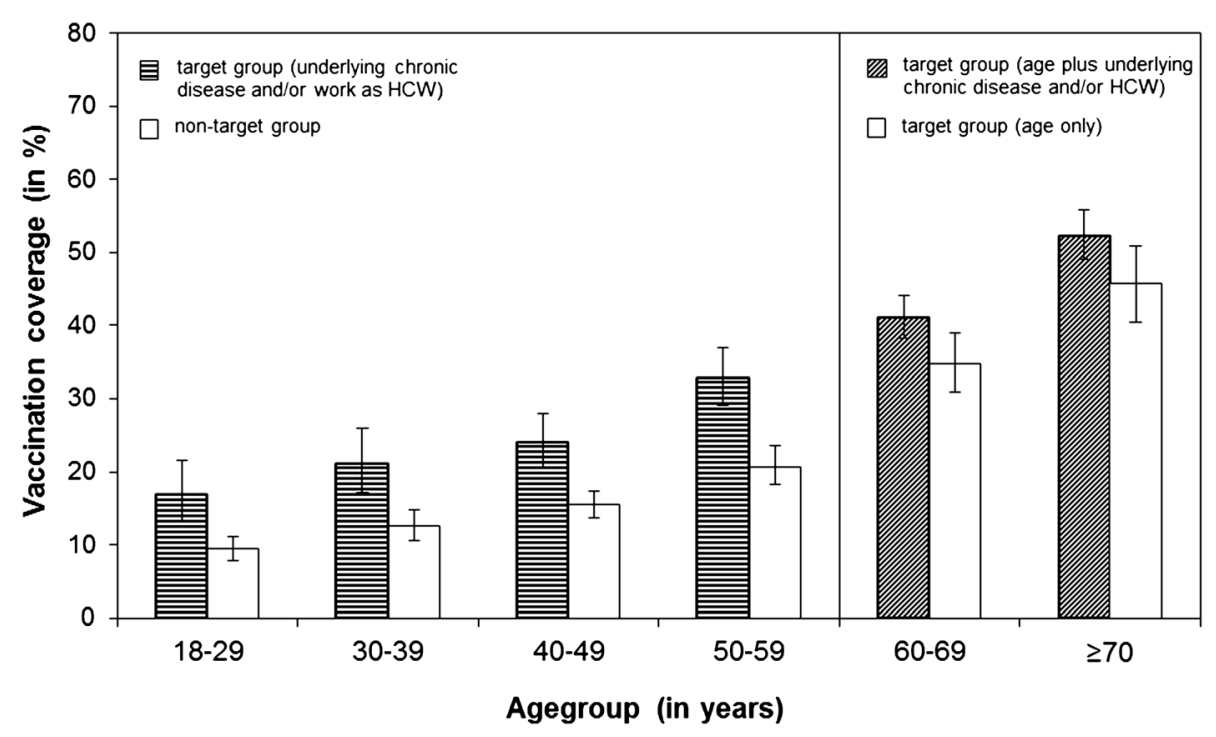

Figure 1 Seasonal vaccination coverage by age group and target group, season 2009/10. 


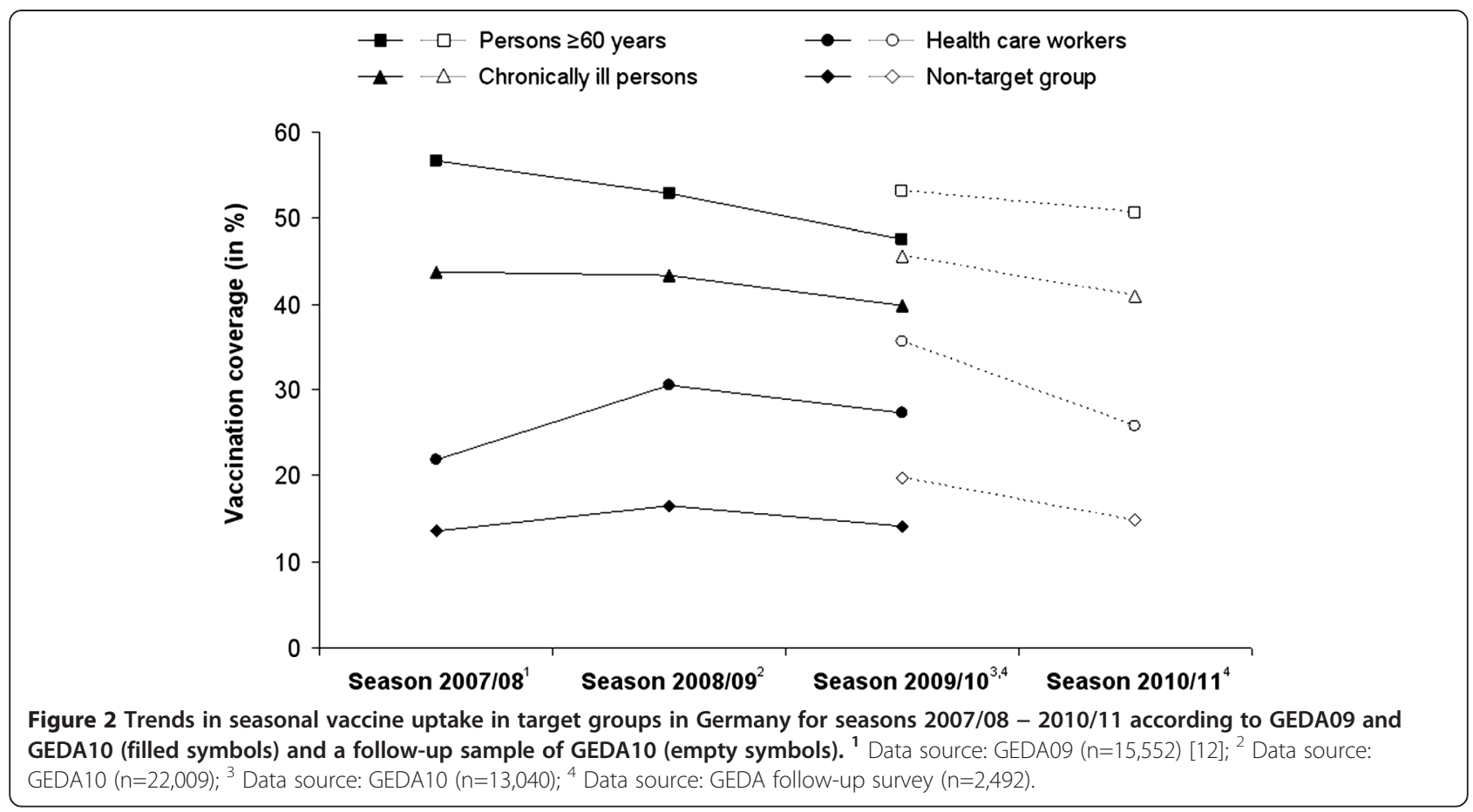

from a large population-representative telephone survey. By using data from a smaller follow-up survey, our study moreover provides the only so far available data on seasonal influenza vaccination coverage in Germany for the post-pandemic season 2010/11. Overall, only $8.8 \%$ of the adult population in Germany followed the official recommendation and received a vaccination against pandemic influenza in season 2009/10. The follow-up survey revealed a decrease in seasonal influenza vaccine uptake in the first post-pandemic season across all target groups when compared to the pre-pandemic season 2008/09, most prominent among $\mathrm{HCW}$. With an average coverage of $50 \%$ in the elderly, $41 \%$ in the chronically ill, and $28 \%$ in HCW in seasons $2008 / 09$ to $2010 / 11$, the EU goal of reaching a seasonal influenza vaccination coverage of at least $75 \%$ in the target groups [20] has not yet been achieved in Germany.

Having received a seasonal influenza shot in the prepandemic season was the strongest predictor for receiving pandemic influenza vaccination in our study. The high correlation between seasonal and pandemic influenza vaccine uptake highlights the significance of habitual behaviour with regard to influenza vaccination decisions. In addition and independent from this factor, persons belonging to at least one of the recommended target groups for seasonal influenza vaccination were significantly more likely to receive a pandemic influenza vaccination than persons not belonging to a target group. Our results are broadly in line with the findings of a prospective monitoring survey on pandemic influenza vaccination in Germany [6] and two reviews investigating determinants of pandemic vaccine uptake $[21,22]$. Prior seasonal vaccination was not only found to be positively associated with the intention to receive the pandemic vaccination among adults in several industrialized countries (e.g. in the UK [23], France [24], Australia [25], and the US [26]) but also with the actual receipt of the vaccination (e.g. $[6,27,28])$. When developing vaccination strategies for future pandemic situations one should therefore consider targeted strategies for enhancing coverage among those who do not fall within the target groups for seasonal influenza vaccination and thus do not regularly receive a seasonal influenza shot. A further opportunity to enhance compliance with national recommendations and therefore vaccination coverage in a future pandemic situation could be to increase seasonal vaccine uptake in the target groups. However, major reasons for not being vaccinated were the perception that vaccination was not necessary or not safe. It can be assumed that both reasons will not be barriers to high pandemic vaccine uptake in a future pandemic setting if the mortality is much higher than during the 2009/10 pandemic.

A higher uptake of seasonal influenza vaccines in season 2009/10 was observed in the follow-up survey population when compared to the total GEDA10 study population in $2009 / 10$. It is therefore very likely that the point estimates for seasonal influenza vaccination coverage for the 2010/11 season, which were based on data from the same follow-up survey, were also overestimated. Taking into consideration that acceptance of seasonal influenza vaccination was higher in the follow-up survey 
Table 3 Determinants of pandemic influenza vaccine uptake, Germany, season 2009/10

\begin{tabular}{|c|c|c|c|c|}
\hline & \multirow[t]{2}{*}{$\mathrm{n}^{\#}$} & \multirow[t]{2}{*}{ A(H1N1)pdm09 vaccination coverage; \% $(95 \% \mathrm{Cl})^{\#}$} & \multicolumn{2}{|c|}{ Odds Ratio $(95 \% \mathrm{Cl})^{\#}$} \\
\hline & & & univariate & multivariable \\
\hline Total & 13,032 & $8.8(8.2-9.5)$ & - & - \\
\hline \multicolumn{5}{|l|}{$\underline{\text { Sex }}$} \\
\hline female & 6,730 & $7.8(7.1-8.7)$ & $1^{\text {ref }}$ & $1^{\text {ref }}$ \\
\hline male & 6,302 & $9.9(8.9-10.9)$ & $1.29(1.14-1.45)^{* *}$ & $1.36(1.15-1.61)^{* *}$ \\
\hline \multicolumn{5}{|c|}{ Vaccinated against seasonal influenza in season $2008 / 09$} \\
\hline \multicolumn{5}{|c|}{ for agegroup $18-59$ years } \\
\hline no & 7,218 & $4.4(3.8-4.9)$ & $1^{\text {ref }}$ & $1^{\text {ref }}$ \\
\hline yes & 1,784 & $22.9(20.7-25.3)$ & $6.54(5.43-7.87)^{* *}$ & $5.98(4.96-7.20)^{* *}$ \\
\hline \multicolumn{5}{|c|}{ for agegroup $\geq 60$ years } \\
\hline no & 1,925 & $1.9(1.3-2.8)$ & $1^{\text {ref }}$ & $1^{\text {ref }}$ \\
\hline yes & 2,078 & $18.5(16.1-21.1)$ & $11.60(7.51-17.01)^{* *}$ & $4.76(1.60-14.18)^{* *}$ \\
\hline \multicolumn{5}{|c|}{ Place of residence } \\
\hline WFS & 10,338 & $8.9(8.2-9.6)$ & $1^{\text {ref }}$ & n.s. \\
\hline EFS & 2,694 & $8.7(7.3-10.3)$ & $0.98(0.84-1.14)$ & \\
\hline \multicolumn{5}{|c|}{ Socioeconomic Status } \\
\hline low & 2,524 & $7.6(6.1-9.5)$ & $1^{\text {ref }}$ & $1^{\text {ref }}$ \\
\hline medium & 7,777 & $8.0(7.3-8.9)$ & $1.06(0.89-1.25)$ & $1.01(0.77-1.33)$ \\
\hline high & 2,688 & $12.2(11.1-13.4)$ & $1.69(1.40-2.03)^{* *}$ & $1.61(1.23-2.11)^{* *}$ \\
\hline \multicolumn{5}{|c|}{ Health care workers } \\
\hline no & 687 & $8.4(7.8-9.1)$ & $1^{\text {ref }}$ & $1^{\text {ref }}$ \\
\hline yes & 12,345 & 16.5 (13.9-19.6) & $2.16(1.75-2.67)^{* *}$ & $2.30(1.78-2.96)^{* *}$ \\
\hline \multicolumn{5}{|c|}{ Underlying chronic disease } \\
\hline no & 8,786 & $7.2(6.6-7.9)$ & $1^{\text {ref }}$ & $1^{\text {ref }}$ \\
\hline yes & 4,246 & $12.2(10.9-13.6)$ & $1.79(1.58-2.02)^{* *}$ & $1.42(1.19-1.69)^{* *}$ \\
\hline
\end{tabular}

${ }^{\#}$ weighted data; ${ }^{*} p<0.05 ;{ }^{*} \mathrm{p}<0.001 ;$ ref $=$ reference category; $n . s .=$ not significant; WFS=Western federal states, EFS=Eastern federal states.

$\mathrm{p}$-value for interaction between agegroup*seasonal influenza vaccination status: 0.011 .

population, our study results suggest that seasonal influenza vaccine uptake in the recommended target groups in Germany has decreased in the post-pandemic season 2010/11, not only in comparison to season 2008/09 but also to the pandemic season 2009/10 (compare Figure 2, empty symbols). Hence, our findings are discordant with observations made in several other industrialised countries. For instance, seasonal influenza vaccination coverage in the UK remained stable between seasons 2009/10 and 2010/11 among at risk persons under 65 years of age (51.6\% vs. $50.4 \%$ vaccination coverage) as well as among persons aged $\geq 65$ years ( $72.4 \%$ vs. $72.8 \%$ ) [29]. Among high-risk persons aged 18-64 years living in the US, seasonal influenza vaccine uptake was $46.2 \%$ in the pandemic and $46.7 \%$ in the post-pandemic season [30]. However, in both countries acceptance and uptake of pandemic influenza vaccination was higher as compared to Germany (UK: $37.6 \%$ vaccination coverage in clinical risk groups [29]; US: $41.2 \%$ among all persons aged $\geq 6$ month [31]). In France, despite the poor uptake of pandemic influenza vaccines (11.1\%), an increase in seasonal influenza vaccine uptake was observed in the post- pandemic season among persons aged $\geq 65$ years with underlying chronic conditions (62.6\% in season 2009/10 [27] vs. $71.0 \%$ in season $2010 / 11$ [32]). In the upcoming years, the uptake of seasonal influenza vaccines should be carefully monitored in Germany in all target groups to identify if this trend continues. Especially the strong decrease in vaccination coverage among HCW is of concern, and communication activities should be strengthened especially for this target group not only to achieve individual protection of this target group but also to protect vulnerable patients managed by HCW.

In our study 'fear of side effects' was found to be the most frequently stated reason for rejecting pandemic vaccination, thereby confirming findings of 13 smaller consecutive surveys carried out during the pandemic in Germany [33]. Conversely, believing that the pandemic vaccine is safe was significantly associated with the receipt of the pandemic vaccine in many countries worldwide [21]. Appropriate addressing of vaccine safety concerns by public health authorities may be an important factor to maintain public trust in national vaccination recommendations 
and beyond that to enhance vaccine uptake in future pandemic situations [22,33].

In our study, $8.5 \%$ of those who did not receive a pandemic influenza vaccination stated that they reject vaccinations in general. This translates into a proportion of $7.7 \%$ for the total study population. Little is known about the exact proportion of vaccination opponents among the general adult population in Germany. In a recent survey performed by the German Federal Centre for Health Education (BZgA) among 3,002 parents of children aged $0-13$ years, $35 \%$ stated that they reject particular vaccinations for their children, but only $1 \%$ of parents reject vaccinations in general [34]. Since our study did not focus on the general rejection of vaccinations in the population, we did not ask further detailed questions related to this topic to verify this attitude and the underlying reasons. Therefore, this figure must be interpreted with caution.

Our study has some limitations that need to be acknowledged. Calculation of influenza vaccination coverage was based on self-reported vaccination status and may therefore be prone to recall problems. However, it was shown in several studies that self-report of influenza vaccination status has an adequate degree of validity $[35,36]$. Furthermore, the response rate in GEDA10 was comparatively low at $29 \%$. However, it should be noted that the chosen method of calculating the response rate (namely Response Rate 3 as defined by AAPOR [18]) is a very conservative approach and that our response rate is comparable to studies using the same approach (e.g. CDC-Behavioral Risk Factor Surveillance Rates Report [37]). Finally, it cannot be ruled out that other reasons than the controversial discussions on the pandemic vaccination have also contributed to the observed drop in seasonal influenza vaccination coverage in $2010 / 11$.

\section{Conclusion}

In conclusion, poor compliance with official vaccination recommendation resulting in low uptake of pandemic influenza vaccines during the pandemic season 2009/10 suggests that public communication strategies and vaccination campaigns during the influenza $\mathrm{A}(\mathrm{H} 1 \mathrm{~N} 1)$ pdm09 pandemic in Germany were not successful. In addition, our results raise concerns that controversial discussions about the safety and necessity of pandemic influenza vaccines may have contributed to decreased seasonal influenza vaccine uptake in the first postpandemic season. It is therefore crucial to develop concerted communication strategies based on the lessons learned from the 2009/10 influenza pandemic and to include them in the national pandemic preparedness plan. This should be done, not only with respect to a competent handling of pandemic situations but also to avoid a decrease in the acceptance of vaccinations in general. In this respect, communication strategies and different modes of communication to specific target groups should be evaluated and implemented already in noncrisis situations, to be enhanced during a pandemic influenza situation or other public health crisis. This is of particular importance, since seasonal influenza vaccine uptake in the recommended target groups in Germany stagnated at a low level since 2005 [38] and does by far not meet the EU goal of 75\% [20]. Further studies should be conducted to monitor the trends of seasonal influenza vaccine uptake in Germany in the specific target groups including pregnant women (which is a target group for seasonal influenza vaccination since 2010) and to precisely identify barriers to influenza vaccination in the upcoming years which might differ from the pandemic and the first post-pandemic season. This information would be crucial to guide public health authorities in developing more effective communication strategies for seasonal influenza vaccination tailored to specific target groups.

\section{Competing interests}

The authors have declared no conflict of interest.

\section{Authors' contributions}

All authors made substantial contributions to the study. SM was involved in the development of the GEDA10 study design and contributed to the Methods section. MMB, DW, and OW developed the study design of the GEDA10 follow-up survey. MMB analysed the data in consultation with DW, GF, OW and GK. MMB wrote the draft version of the manuscript. All authors have read, carefully reviewed and approved the final version of the manuscript.

\section{Acknowledgements}

We would like to thank all colleagues at the Robert Koch Institute who were involved in the planning and implementation of the GEDA2010 and the GEDA follow-up survey.

\section{Author details}

${ }^{1}$ Immunization Unit, Robert Koch Institute, Berlin, Germany. ${ }^{2}$ Department for Infectious Disease Epidemiology, Robert Koch Institute, Berlin, Germany. ${ }^{3}$ Department of Epidemiology and Health Reporting, Robert Koch Institute, Berlin, Germany. ${ }^{4}$ Charité - University Medicine Berlin, Berlin, Germany. ${ }^{5}$ Department for Infectious Disease Epidemiology, Immunization Unit, Robert Koch Institute, DGZ-Ring 1, Berlin 13086, Germany.

Received: 9 June 2012 Accepted: 22 October 2012

Published: 31 October 2012

\section{References}

1. Robert Koch Institute: Estimation of Influenza-associated deaths during the influenza season 2008/09 and the pandemic season 2009/10. Epidemiologisches Bulletin 2011, 10:75-77.

2. Robert Koch Institute: Report on the epidemiology of influenza in Germany, season 2010/11. 2011, Available at: http://influenza.rki.de/ Saisonberichte/2010.pdf (day of access: 01/02/2012).

3. Fiore $A E$, Uyeki TM, Broder K, Finelli $L$, Euler GL, Singleton JA, Iskander JK, Wortley PM, Shay DK, Bresee JS, et al: Prevention and control of influenza with vaccines: recommendations of the Advisory Committee on Immunization Practices (ACIP), 2010. MMWR Recommendations and reports: Morbidity and mortality weekly report Recommendations and reports / Centers for Disease Control 2010, 59(RR-8):1-62. 
4. Robert Koch Institute: Recommendations of the German Standing Committee on Vaccination (STIKO)/July 2011. Epidemiologisches Bulletin 2011, 30:275-294.

5. Robert Koch Institute: Announcement of the Standing Committee of Vaccination (STIKO): Vaccination against Novel Influenza A (H1N1). Epidemiologisches Bulletin 2009, 41:403-426.

6. Walter D, Böhmer MM, Heiden M, Reiter S, Krause G, Wichmann O: Monitoring pandemic influenza $\mathrm{A}(\mathrm{H} 1 \mathrm{~N} 1)$ vaccination coverage in Germany 2009/10 - results from thirteen consecutive cross-sectional surveys. Vaccine 2011, 29(23):4008-4012.

7. Krause G, Gilsdorf A, Becker J, Bradt K, Dreweck C, Gartner B, Lower J, Marcic A, Nicoll A, Pott $E$, et al: First exchange of experiences concerning the H1N1 pandemic in Germany 2009/2010: report on a workshop held March 22-23, 2010, in Berlin. Bundesgesundheitsblatt Gesundheitsforschung Gesundheitsschutz 2010, 53(5):510-519.

8. Poland GA: Pandemic 2009-2010 influenza vaccine: six lessons learned and the way forward (Allegro not Adagio). Vaccine 2011, 29(4):613-614

9. Poland GA: The 2009-2010 influenza pandemic: effects on pandemic and seasonal vaccine uptake and lessons learned for seasonal vaccination campaigns. Vaccine 2010, 28(Suppl 4):D3-D13.

10. Sammon CJ, McGrogan A, Snowball J, de Vries CS: Factors associated with uptake of seasonal and pandemic influenza vaccine among clinical risk groups in the UK: an analysis using the General Practice Research Database. Vaccine 2012, 30(14):2483-2489.

11. Feufel MA, Antes $G$, Gigerenzer $G$ : Competence in dealing with uncertainty: lessons to learn from the influenza pandemic (H1N1) 2009 Bundesgesundheitsblatt Gesundheitsforschung Gesundheitsschutz 2010, 53(12):1283-1289.

12. Böhmer MM, Walter $D$, Müters $S$, Krause G, Wichmann O: Seasonal influenza vaccine uptake in Germany 2007/2008 and 2008/2009: results from a national health update survey. Vaccine 2011, 29(27):4492-4498.

13. Böhmer MM, Walter D, Krause G, Müters S, Gösswald A, Wichmann O: Determinants of tetanus and seasonal influenza vaccine uptake in adults living in Germany. Hum Vaccin 2011, 7(12):1317-1325.

14. Wiese-Posselt M, Leitmeyer K, Hamouda O, Bocter N, Zollner I, Haas W Ammon A: Influenza vaccination coverage in adults belonging to defined target groups, Germany, 2003/2004. Vaccine 2006, 24(14):2560-2566.

15. Rehmet S, Ammon A, Pfaff G, Bocter N, Petersen LR: Cross-sectional study on influenza vaccination, Germany, 1999-2000. Emerg Infect Dis 2002, 8(12):1442-1447.

16. Kurth BM, Lange $C$, Kamtsiuris $\mathrm{P}$, Holling $\mathrm{H}$ : Health monitoring at the Robert Koch Institute. Status and perspectives. Bundesgesundheitsblatt Gesundheitsforschung Gesundheitsschutz 2009, 52(5):557-570.

17. German Federal Statistical Office: Microcensus. 2008. Available at: www.destatis.de (day of access: 01/05/2012)

18. American Association for Public Opinion Research (AAPOR): Standard Definitions - Final Dispositions of Case Codes and Outcome Rates for Surveys. 2011. Available at: http://www.aapor.org/AM/Template.cfm?

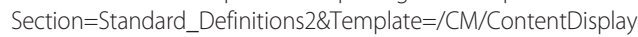
cfm\&ContentID=3156 (day of access: 01/02/2012).

19. Lampert T, Kroll LE: Measurement of socioeconomic status in socialepidemiological studies. In Health inequalities - theories, concepts, and methods. Edited by Richter M, Hurrelmann K. Wiesbaden: Verlag für Sozialwissenschaft: 2006:297-319.

20. Council of the European Union: Council Recommendation of 22 December 2009 on seasonal influenza vaccination. Off J Eur Union 2009, L348:71-72.

21. Brien S, Kwong JC, Buckeridge DL: The determinants of 2009 pandemic A/H1N1 influenza vaccination: a systematic review. Vaccine 2012, 30(7):1255-1264

22. Bish A, Yardley L, Nicoll A, Michie S: Factors associated with uptake of vaccination against pandemic influenza: a systematic review. Vaccine 2011, 29(38):6472-6484.

23. Myers $L B$, Goodwin R: Determinants of adults' intention to vaccinate against pandemic swine flu. BMC Publ Health 2011, 11(1):15.

24. Setbon $M$, Raude J: Factors in vaccination intention against the pandemic influenza A/H1N1. Eur J Public Health 2010, 20(5):490-494.

25. Eastwood K, Durrheim DN, Jones A, Butler M: Acceptance of pandemic (H1N1) 2009 influenza vaccination by the Australian public. Med J Aust 2010, 192(1):33-36.
26. Horney JA, Moore Z, Davis M, MacDonald PD: Intent to receive pandemic influenza $\mathrm{A}(\mathrm{H} 1 \mathrm{~N} 1)$ vaccine, compliance with social distancing and sources of information in NC, 2009. PLoS One 2010, 5(6):e11226.

27. Vaux S, Van Cauteren D, Guthmann JP, Le Strat Y, Vaillant V, de Valk H, Levy-Bruhl D: Influenza vaccination coverage against seasonal and pandemic influenza and their determinants in France: a cross-sectional survey. BMC Publ Health 2011, 11:30.

28. Rodriguez-Rieiro C, Esteban-Vasallo MD, Dominguez-Berjon MF, Astray-Mochales J, Iniesta-Fornies D, Barranco-Ordonez D, Cameno-Heras M, Jimenez-Garcia R: Coverage and predictors of vaccination against 2009 pandemic H1N1 influenza in Madrid, Spain. Vaccine 2011, 29(6):1332-1338

29. Health Protection Agency: Influenza vaccination uptake monitoring on behalf of the Department of Health. 2011. Available at: http://www.hpa.org.uk/web/ HPAweb\&HPAwebStandard/HPAweb_C/1195733756886\#r5 (day of access: 28/03/2012)

30. Centres for Disease Control and Prevention: Recent Influenza Vaccination Trends across Influenza seasons. 2011. Available at: www.cdc.gov/flu/ professionals/vaccinations/trends-summary.htm (day of access: 28/03/2012).

31. Centres for Disease Control and Prevention: Final estimates for 2009-10 Seasonal Influenza and Influenza A(H1N1) 2009 Monovalent Vaccination Coverage - United States, August 2009 through May, 2010. 2011. Available at: www.cdc.gov/flu/professionals/vaccination/coverage_0910estimates.htm (day of access: 28/03/2012).

32. Guthmann JP, Fonteneau L, Bonmarin I, Levy-Bruhl D: Influenza vaccination coverage one year after the $\mathrm{A}(\mathrm{H} 1 \mathrm{~N} 1)$ influenza pandemic, France, 2010-2011. Vaccine 2012, 30(6):995-997.

33. Walter D, Böhmer M, Reiter S, Krause G, Wichmann O: Risk perception and information-seeking behaviour during the 2009/10 influenza $A(H 1 N 1)$ pdm09 pandemic in Germany. Euro Surveill 2012, 17(13):pii:20131.

34. German Federal Centre for Health Education (BZgA): Parental Survey on "Childhood Vaccinations". 2011. Available at: http://www.health-record.net/ resources/Elternstudie_Impfen_im_Kindesalter_2011.pdf (day of access: 01/10/2012)

35. Mangtani $P$, Shah A, Roberts JA: Validation of influenza and pneumococcal vaccine status in adults based on self-report. Epidemiol Infect 2007, 135(1):139-143.

36. Mac Donald R, Baken L, Nelson A, Nichol KL: Validation of self-report of influenza and pneumococcal vaccination status in elderly outpatients. Am J Prev Med 1999, 16(3):173-177.

37. Centres for Disease Control and Prevention: Risk Factor Surveillance System: 2009 Summary Data Quality Report. 2010. Available at: ftp://ftp.cdc.gov/pub/ Data/Brfss/2009_Summary_Data_Quality_Report.pdf (day of access: 01/021/2012).

38. Blank PR, Schwenkglenks M, Szucs TD: Disparities in influenza vaccination coverage rates by target group in five European countries: trends over seven consecutive seasons. Infection 2009, 37(5):390-400.

\section{doi:10.1186/1471-2458-12-938}

Cite this article as: Böhmer et al:: Barriers to pandemic influenza vaccination and uptake of seasonal influenza vaccine in the post-pandemic season in Germany. BMC Public Health 2012 12:938.

\section{Submit your next manuscript to BioMed Central and take full advantage of:}

- Convenient online submission

- Thorough peer review

- No space constraints or color figure charges

- Immediate publication on acceptance

- Inclusion in PubMed, CAS, Scopus and Google Scholar

- Research which is freely available for redistribution 